\title{
Evaluation on Distribution Characteristics of Pore Water Pressure within Saturated Pavement Structure Based on the Proposed Tire-Fluid-Pavement Coupling Model
}

\author{
Binshuang Zheng $\mathbb{D}^{1},{ }^{1}$ Xiaoming Huang $\mathbb{D}^{2},{ }^{2}$ Jingwen $M a,{ }^{2}$ Zhengqiang Hong, \\ Jiaying Chen, ${ }^{2}$ Runmin Zhao, ${ }^{2}$ and Shengze $\mathrm{Zhu}^{2,3}$ \\ ${ }^{1}$ School of Modern Posts, Nanjing University of Posts and Telecommunications, Nanjing 210003, China \\ ${ }^{2}$ School of Transportation, Southeast University, Nanjing 211189, China \\ ${ }^{3}$ Shanghai Jiushi (Group) CO., Ltd., Shanghai 200021, China
}

Correspondence should be addressed to Xiaoming Huang; huangxm@seu.edu.cn

Received 3 June 2020; Accepted 19 June 2021; Published 28 January 2022

Academic Editor: Akbar Heidarzadeh

Copyright (c) 2022 Binshuang Zheng et al. This is an open access article distributed under the Creative Commons Attribution License, which permits unrestricted use, distribution, and reproduction in any medium, provided the original work is properly cited.

\begin{abstract}
To investigate the flow characteristics of pore water in asphalt pavement and the variation law of the pore water pressure under vehicle loading, a novel method based on BA network and quartet structure generation set method was proposed to reconstruct the three-dimensional (3D) pavement model with pores. The permeability coefficient and the gradation curve were adopted to evaluate the reliability and stability of the random growth pavement model. Then, the tire-fluid-pavement coupling model was established with FLUENT 3D based on the fluid Mie-Gruneisen state equation. According to the built fluid-solid coupling model, the pressure-velocity coupled finite volume algorithm was applied to study the distribution of the pore water pressure in asphalt pavement. Results show that the pore water pressure in asphalt pavement decays periodically with time under vehicle loading. For different types of asphalt pavement, the pore water pressure in open-graded friction course (OGFC) pavement is the smallest during the whole process. Moreover, the peak values of the pore water pressure decrease in the order of asphalt concrete (AC) pavement, stone mastic asphalt (SMA) pavement, and OGFC pavement. The maximum negative value of the pore water pressure is generally less than 0.3 times the maximum positive values. As for saturated pavement pores, the pore water pressure is hardly affected by the water film thickness. The positive peak value of the pore water pressure increases on an approximate parabolic curve as the vehicle speed improves gradually, while the negative one remains largely unchanged. The results are expected to help reduce tire hydroplaning risk and provide guidance for the selection of asphalt mixtures of drainage asphalt pavement.
\end{abstract}

\section{Introduction}

Due to its advantages of smoothness, low noise, riding comfort, and so on, especially, the drainage capacity caused by the existence of internal pores, the asphalt pavement becomes a typical pavement in the rainy areas of southern China. On rainy days, rainwater will infiltrate into the pavement internal structure via the connected pores and cracks. Under the repeated action of vehicle loading, the pore water pressure usually generates within asphalt pavement internal structure, which seriously damages the adhesion between asphalt binder and aggregates [1, 2]. Furthermore, it can cause moisture damage and even structural damage of asphalt pavement [3-6]. The pore water pressure in asphalt pavement is considered as one of the major causes of pavement damage. Studies have shown that asphalt pavement with porosity between $8 \%$ and $15 \%$ is more prone to water damage, which is directly related to the pore water pressure [7].

In order to study the change law of pore water pressure in asphalt pavement, studies about numerical calculations and theoretical analyses have been carried out to calculate 
the pore water pressure [8-13]. For example, based on the porous media theory and Biot theory, Sun et al. [14] built a hydromechanical coupling model to investigate the spatial distribution and the change law with time of pore water pressure in asphalt pavement. Existing research results indicate that there is a positive correlation between the pore water pressure and vehicle speed based on the fluid dynamics theory. However, most of the research could not directly reflect the pore water flow characteristics in pavement [15-21]. To investigate the flow characteristics of pore water directly, the three-dimensional (3D) reconstruction of asphalt pavement with actual pore structure becomes feasible and important in pavement research field. Some reconstruction methods were proposed by researchers, such as CT scanning, microscope, and image technology [22-24]. Generally, the asphalt pavement texture can be obtained by microscope to reconstruct the 3D model of asphalt pavement surface. Thus, some scholars have reconstructed the 3D model of pavement according to the tomographic image of the asphalt mixture specimen scanned by CT technology, such as Wang et al. [25], Kutay et al. [26], and Ran et al. [27]. However, the experiment methods mostly have disadvantages of low accuracy, long time, and certain error. In addition, some researchers used a simplified model to study the hydrodynamic pressure characteristics with the mechanical characteristics, but it cannot reflect the real situation of the water flowing from the asphalt pavement void when the tire is subjected to hydroplaning $[28,29]$. Furthermore, the CT scanning technology and image acquisition work for the reconstruction of asphalt pavement model always cost much time and money, especially, the measurement precision is usually low due to testing equipment. The reconstructed asphalt pavement model also lacks randomness, which does not reflect real pavement structures.

According to the research shortcomings mentioned above, the random generation methods of the pavement are gradually developed, especially the random aggregate delivery method and the quartet structure generation set (QSGS) method are mainly adopted. In general, the random aggregate delivery method is more in accordance with the engineering practice, and it can simulate the aggregate formation and screening and forming process of the asphalt mixture [30]. Besides, the QSGS method can quantitatively describe the structure of porous media from the microscopic aspect, which is more suitable for studying the pore hydrodynamic pressure in asphalt pavement. For example, in order to reflect the random distribution characteristics of most porous media, Wang et al. [31] proposed an internal morphological random growth method based on random growing cluster theory, that is, the QSGS method. The porous medium produced by the QSGS method is close to the real microscopic structure. Based on the QSGS method, Li et al. [32] reconstructed the clay microstructure that could be directly imported into the finite element software. When the asphalt pavement model is constructed by the QSGS method, the growth mode is close to the formation process of the porous medium, and the growth rate is quick and the pore distribution is uniform. However, the gradation curve characteristics of the asphalt concrete itself cannot be well reflected. Nevertheless, the BA network model is a typical complex network model established by Albert-László Barabási and Réka Albert. This model has the characteristics of preferential connectivity, growth, and scale-free. Thus, it is easier to produce aggregation effect by using such a network form [33]. Therefore, on the basis of the QSGS method, we tried to apply the BA network model to further control the growth of the pores, so that the pore distribution and geometric construction of the randomly growth asphalt pavement are more consistent with the actual situation.

Taking the above research status into consideration, in order to investigate the pore water pressure within asphalt pavement internal structure under repeated vehicle loading on rainy days, the $3 \mathrm{D}$ model of asphalt pavement based on the BA network and QSGS method was reconstructed in this study. Also, permeability coefficient and gradation curve were selected to evaluate the reliability of the random growth pavement model. Then, based on the fluid-solid coupling theory, the tire-water-pavement coupling model was established by FLUENT 3D program. The change rule of pore water pressure under moving traffic loads and the influence of the pavement type, water film thickness, and vehicle speed on the pore water pressure were also analyzed.

\section{Research Outline}

In this study, a method based on BA network and QSGS method for 3D model reconstruction of asphalt pavement with pores was proposed to investigate the pore water pressure distribution in pavement internal structure under repeated traffic loads. The objectives and scope of this study are as follows:

(1) The primary objective of this study is to determine the pore water flow characteristics in asphalt pavement according to the numerical method and simulation modeling. Based on the BA network and QSGS method, 3D random growth model of asphalt pavement was constructed. Then, two indexes of permeability coefficient and gradation curve are used to evaluate the reliability of random growth pavement models.

(2) The established asphalt pavement model and the tire rolling model are introduced into the FLUENT 3D program to establish the tire-water-pavement coupling model. The unidirectional fluid-solid coupling algorithm is adopted to ensure the calculation efficiency. The free surface of water flow is constructed on the water-solid coupling interfaces to transfer the pressure.

(3) Based on the coupled tire-water-pavement model, the pressure-velocity coupled finite volume algorithm is applied to increase the calculation speed for model convergence to obtain the pore hydrodynamic pressure curve varying with time. We analyzed the effects of relevant factors on the pore hydrodynamic pressure of the asphalt pavement, such as asphalt pavement type, water film thickness, and driving speed. 


\section{Reconstruction of Asphalt Pavement with Pores}

3.1. Quartet Structure Generation Set. The quartet structure generation set (QSGS) method is a probabilistic algorithm for randomly growing pores. Taking the construction of twodimensional solid particles and pores as an example, regard the pores as growth phases and solid particles as non-growth phases, set the initial solid particles as randomly distributed, and grow randomly according to a certain probability. The specific construction steps are as follows:

(i) Step 1. Construct a random growth region, in which the growth nucleus of the random pore is preset. Set the growth nuclear distribution probability as $p_{\mathrm{d}}$. Meanwhile, the growth nucleus is used to define the initial node of the pore growth.

(ii) Step 2. Take the randomly distributed pore growth nucleus in Step 1 as starting point and make them randomly grow in eight directions with the probability of $P_{i}$, as shown in Figure 1(a).

(iii) Step 3. Make any individual pore node in each growth direction having a probability of $p_{\mathrm{i}}$ to grow into a new pore node in a certain direction. If the new growth node itself is a pore in a certain direction, skip it and continue to grow in different directions.

(iv) Step 4. Repeat Step 2 and Step 3 until the porosity reaches the initial value $n$. Then, a two-dimensional asphalt pavement with pore distribution is obtained, as shown in Figure 1(b).

The random aggregate delivery method can be utilized to simulate the aggregate formation and screening and forming process of asphalt mixture. However, it is difficult to accurately characterize the contact state between aggregates, and it always takes too much time. Compared with random aggregate delivery method, the QSGS method can quantitatively describe the structure of the porous medium from a microperspective, and its growth mode is close to that of porous media. This growth mode is suitable for the semirandom generation of the skeleton, mortar, and asphalt components for the asphalt concrete pavement. Moreover, the distribution of asphalt mortar has a certain regularity and the formation speed is fast. Therefore, the QSGS method will be used to reconstruct the asphalt pavement.

3.2. BA Scale-Free Networks. When constructing the BA scale-free network model, the number of initial model nodes is defined as $m_{0}$, and each initial node can be connected to other nodes with the number of $n$. According to the set time step, the new node continues to grow on the basis of node $m_{0}$. Also, the probability $\amalg\left(k_{i}\right)$ of adding a new node $v_{i}$ to the existing node in the network is expressed as follows:

$$
\prod\left(k_{i}\right)=\frac{k_{i}}{\sum_{1 \leq j \leq N} k_{j}}, \quad 1 \leq i \leq N,
$$

where $k_{i}$ is the degree of node $v_{i}, N$ is the total number of nodes, and $\sum k_{j}$ is the total number of degrees. Assuming that the number of initial nodes $m_{0}$ is 3 , its evolution can be represented by Figure 2 .

The nature of asphalt mixture image has certain similarity with BA scale-free network model: it essentially is a semi-randomly generated pixel point set. Such a semirandomly generated set of pixel points adjacent to the aggregate pixel point group is more likely to be an aggregated pixel point in terms of probability. That is to say, a pixel point adjacent to the pore pixel group is more likely to be a pore pixel point in terms of probability.

\subsection{Random Generation Algorithm of 3D Asphalt Pavement} Model. Referring to the traditional QSGS method to construct the asphalt pavement, the BA scale-free network model was applied to further control the growth of the pores, so that the pore distribution and geometric construction of the asphalt pavement are consistent with the actual state. In this study, the aggregate phase is set as the growth phase, and the specific steps of reconstructing a 3D random growth model of asphalt pavement are as follows:

(i) Step 1. Construct a cuboid region for pore growth. The pixel of the growth region size is set as $1200 \times 678 \times 223$ with each pixel size of $0.4 \mathrm{~mm} \times 0.4 \mathrm{~mm} \times 0.4 \mathrm{~mm}$. The aggregate growth nucleuses are randomly distributed in the constructed region with the distribution probability of 0.1 . That is, about $10 \%$ of the regions are preset as aggregate pixel points.

(ii) Step 2. Traverse the pixels in the cuboid region gradually from top to bottom to calculate the estimated diameter of each unknown pixel. Then, the probability of the unknown pixel being the aggregate pixel is analyzed. Meanwhile, the unknown pixel is determined as the aggregate pixel or not according to the roulette algorithm.

(iii) Step 3. Remove $5 \%$ of the aggregate pixels randomly after three traversals to increase the randomness and variability of the model.

(iv) Step 4. Repeat Step 2 and Step 3 to continuously traverse the growing aggregate pixels for the generated area until the number of aggregated pixels reaches the total number of pixels in the cuboid region constructed by pictures.

Based on the above steps, randomly generated $3 \mathrm{D}$ asphalt concrete (AC) pavement was reconstructed with MATLAB software programming, and the specific process is as follows:

(i) The dynamic planning algorithm is adopted to calculate the optimal image of the AC asphalt mixture image diameter by CT scanning technology. At the same time, the pixels of the scanned images are extracted as the judgment basis.

(ii) Initialize the model and aggregate distribution and store the data in the three-dimensional matrix. 


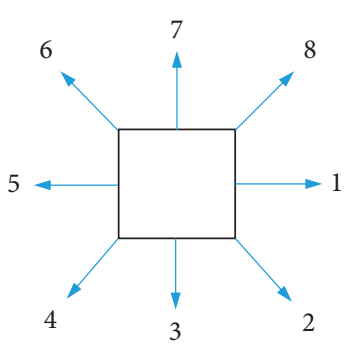

(a)

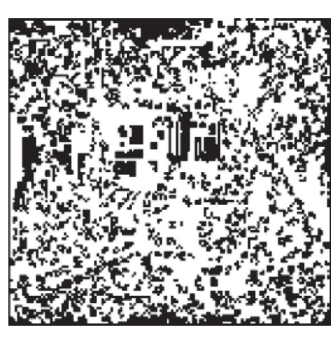

(b)

FIGURE 1: 2D model of asphalt pavement based on QSGS method. (a) Random growth in different directions. (b) 2D model with random pore distribution.

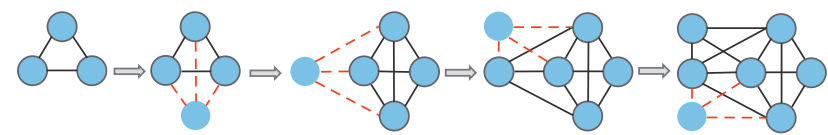

FIGURE 2: Evolution process when the number of initial nodes is 3.

Then, take the existing clusters to be divided or aggregated.

(iii) Traverse and fit the geometry construction from different directions, use the roulette algorithm to determine the aggregate location, and let it grow randomly. During the growth process, randomly remove pixels to maintain the randomness of the generated road surface.

(iv) The random generation process of pavement is actually a semi-random process. The main idea is to identify the asphalt pixels in CT scanning images of asphalt mixture. Based on this, the pixels are randomly grown in the preset area to simulate the $3 \mathrm{D}$ asphalt pavement.

(v) Based on the above steps, the random generation process of stone mastic asphalt (SMA) pavement and open-graded friction course (OGFC) asphalt pavement is similar. After identifying the CT scanning images of SMA asphalt mixture or OGFC asphalt mixture, the pixel cluster diameters are counted and its pixel characteristics are identified to be judgment basis for the random growth of the pavement model.

(vi) Thereafter, the reconstructed 3D models get meshed, mirrored, and expanded, and then the randomly generated 3D model for AC pavement, SMA pavement, and OGFC pavement was obtained. Taking the meshed 3D AC asphalt pavement model as example, we imported it into Fluid 3D mechanics software as shown in Figure 3.

3.4. Validation of $3 D$ Asphalt Pavement Model. The randomly generated model of asphalt pavement is different in each operation. Thus, the permeability coefficient and gradation curve were selected to verify the reliability of the randomly generated model of asphalt pavement in this study. In this section, AC pavement was taken as research project to validate the reliability and accuracy of the randomly generated model.

3.4.1. Permeability Coefficient of Asphalt Mixture. The permeability coefficient is an important indicator to reflect the permeability of asphalt pavement. Usually, the constant water head penetration test was applied to measure the permeability coefficient. The 3D model of asphalt pavement based on CT scanning technology and the random growth pavement model based on BA network and QSGS method were introduced into the fluid mechanics program. Then, the permeability coefficient $k$ of asphalt pavement was numerically calculated based on the following steps:

(i) Step 1. Number the above 3D reconstructed AC asphalt pavement model by CT scanning technology as $\mathrm{A}$. Then, randomly generate models of above asphalt pavement three times to obtain the 3D pavement model, which are numbered as $B_{1}, B_{2}$, and $B_{3}$, respectively. Furthermore, the above four models are imported into fluid dynamics program.

(ii) Step 2. Set the water flow as the $k-\varepsilon$ turbulence model and the water film thickness on road surface as $2 \mathrm{~mm}$. Keep water film thickness constant to simulate the constant water head. The model bottom is set as the wall boundary, the periphery of the model is set as the outflow boundary, and the water inlet is set as the pressure boundary conditions with $0 \mathrm{kPa}$.

(iii) Step 3. Assume that the water flow covers the whole pavement model so that the pavement model can be initially saturated.

(iv) Step 4. Calculate the water output within $1 \mathrm{~s}$ of the above four models under the constant head condition. The permeability coefficients of the four reconstructed models are calculated according to equation (2), and the results are shown in Table 1.

$$
k=\frac{V L}{A \cdot \Delta h \cdot t},
$$

where $V$ is the flow volume, $L$ is the height, $A$ is the crosssectional area, $\Delta h$ is head difference between the section and water film, and $t$ is drainage time. 


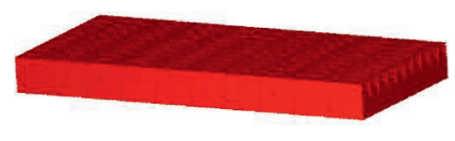

(a)

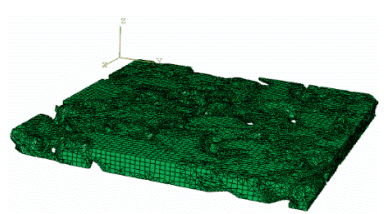

(b)

FIgURE 3: Reconstruction of AC pavement model with different methods. (a) QSGS method combined with BA networks. (b) CT scanning technology.

TABLE 1: Permeability coefficient of asphalt pavement models.

\begin{tabular}{lcccc}
\hline Models & $\mathrm{A}$ & $\mathrm{B}_{1}$ & $\mathrm{~B}_{2}$ & $\mathrm{~B}_{3}$ \\
\hline Permeability coefficient $(\mathrm{cm} / \mathrm{s})$ & 0.0169 & 0.0162 & 0.0171 & 0.0168 \\
\hline
\end{tabular}

From Table 1, the relative error of permeability coefficient among the $3 \mathrm{D}$ reconstructed pavement $\mathrm{A}$ and the three randomly generated $3 \mathrm{D}$ pavements is $1.18 \%$, which is within the range of the allowable error. It can be seen that the randomly generated pavement model is consistent with the real $3 \mathrm{D}$ reconstructed model. Therefore, the randomly generated models have certain stability.

3.4.2. Gradation Curve of Asphalt Mixture. Aggregates with different particle sizes are matched according to a certain ratio to obtain higher compactness or strength, that is, mixture gradation. The gradation curve can reflect the essence of asphalt mixture. In this study, the following method was used to estimate the gradation of the randomly generated 3D model of asphalt pavement.

To obtain the equivalent diameter of aggregate where the pixel A is located, it is necessary to acquire the number of consecutive aggregate pixels at the left, right, upper, and lower sides of the pixel point A. In Figure 4, it shows that the number of consecutive pixels on the four sides is $1,3,2$, and 2 , respectively. The diameter in the left and right directions is 4 , and that in the up and down directions is 4 . Then, the harmonic mean value is determined. The aggregate equivalent diameter of pixel point $\mathrm{A}$ is 4 at the moment. In order to increase the calculation accuracy of the aggregate equivalent particle size, the aggregate equivalent diameter is also calculated from the diagonal direction in this study. Thereafter, the two calculation results are subjected to harmonic averaging calculation. The profiles of the 3D randomly generated asphalt pavement model are cut into sections to obtain the equivalent diameter of the aggregates. Finally, the number of pixels within the sieve range is counted and converted into a gradation curve.

Based on the above specific method, the gradation curves of $3 \mathrm{D}$ randomly generated models of $\mathrm{B}_{1}, \mathrm{~B}_{2}$, and $\mathrm{B}_{3}$ are compared with the A model (that is $\mathrm{AC}$ asphalt mixture), and the results are shown in Table 2.

From Table 2, the gradation curves of the pavement models based on randomly generated method tend to be consistent with those of the actual AC asphalt mixture. Compared with the randomly generated model method with CT technology, the largest relative error is $2.62 \%$, which is within the range of allowable error of $5 \%$. It shows that the

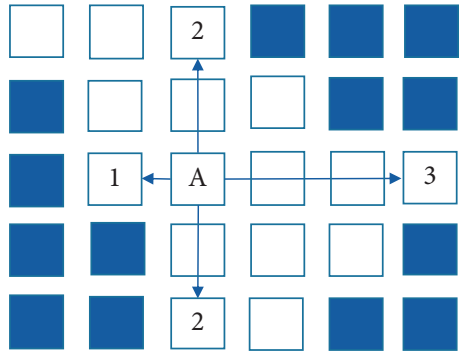

FIGURE 4: Gradation calculation of the random growth model.

randomly generated model of the asphalt pavement has certain reliability and accuracy. Furthermore, the gradation curves of three kinds of randomly generated pavement models vary consistently indicating that the randomly generated model has significant stability.

\section{Tire-Fluid-Pavement Coupling FE Model}

4.1. Patterned Inflatable Tire Modeling. Based on the achievement of the research group [34], the 205-55-R16 tire model is adopted as the research object, in which the tire rubber material adopts the Yeoh constitutive model [35] to simulate the mechanical properties of the rubber superelastic material. As for the rubber-cord composite material containing the reinforcement material, the surface unit embedded the Rebar units is used to simulate the skeleton material passing through the rubber material in the tire structure. The specific material parameters can be obtained by referring to the literatures $[36,37]$.

In order to study the hydrodynamic pressure distribution and the water flow characteristics in the complex pores of the asphalt pavement during the tire rolling process, the tire rolling model is selected in this paper. In view of the slight deformation of the tire rim and the road surface, the rim and the pavement are regarded as a rigid surface. Also, the rim is formed by analytical rigid body to ensure that the rim is consistent with the tire center. The augmented Lagrangian method is applied to describe the contact behavior between the rim and the tire.

4.2. Fluid Modeling. The established asphalt pavement model and the gridded tire model are introduced into the FLUENT 3D software to establish the tire-water-pavement coupling model. Among them, the flow state model is adopted for the water flow region, which reflects the relationship between the fluid density $\rho$, the pressure $p$, and the internal energy $E_{\mathrm{m}}$, as 
TABLE 2: Gradation curves of 3D randomly generated models and AC asphalt mixture.

\begin{tabular}{|c|c|c|c|c|c|c|c|c|c|c|}
\hline \multirow{3}{*}{ Specimens } & & \multirow{2}{*}{\multicolumn{9}{|c|}{$\begin{array}{l}\text { Sieve diameter } \\
\text { Pass rate }(\%)\end{array}$}} \\
\hline & & & & & & & & & & \\
\hline & & 0.15 & 0.3 & 0.6 & 1.18 & 2.36 & 4.75 & 9.5 & 13.2 & 16 \\
\hline \multirow{5}{*}{$3 \mathrm{D}$ randomly generated models } & & 8.9 & 13.2 & 17.9 & 26.8 & 36.1 & 52.7 & 76.5 & 94.3 & 100 \\
\hline & $\mathrm{B}_{1}$ model & 9.2 & 13.7 & 18.3 & 27.4 & 36.9 & 52.8 & 79.1 & 97.8 & 100 \\
\hline & $\mathrm{B}_{2}$ model & 8.1 & 12.6 & 16.8 & 25.9 & 35.7 & 49.9 & 72.2 & 90.1 & 100 \\
\hline & $\mathrm{B}_{3}$ model & 8.7 & 12.5 & 17.4 & 26.3 & 35.3 & 51.6 & 75.9 & 93.9 & 100 \\
\hline & Average value & 8.67 & 12.93 & 17.50 & 26.53 & 35.97 & 51.43 & 75.73 & 93.93 & 100 \\
\hline Relative error (\%) & & 2.62 & 2.02 & 2.23 & 1.00 & 0.37 & 2.40 & 1.00 & 0.39 & 0 \\
\hline
\end{tabular}

shown in Figure 5(a). The state equation is applied to describe the impact effect of the water flow, and the dynamic response of the water flow is obtained by solving the equation of motion of the liquid particles under the impact effect. Thus, considering the flow characteristics of water film under the impact effect of high-speed tire, combined with the superiority of the state equation in simulating impact explosion, the high-pressure state equation in solid form is used in this study. The Mie-Gruneisen state equation is selected to represent the state of water flow, which is expressed as follows:

$$
p=p_{H}\left(\frac{1-\Gamma_{0} \eta}{2}\right)+\Gamma_{0} \rho_{0} E_{m}
$$

where $p$ is the pressure which the water flow is subjected to, $p_{H}$ is the Hugoniot impact pressure, $s$ and $\Gamma_{0}$ are the material constants, $\rho_{0}$ is the initial density of the water flow, $\rho$ is the density after impact effect, $\eta=1-\rho_{0} / \rho$, and $E_{\mathrm{m}}$ is the ratio of internal energy of water. The fitting form of flow Hugoniot impact curve is obtained by the conservation of mass and momentum, as shown in the following equation:

$$
p_{H}=\frac{\rho_{0} c_{0}^{2} \eta}{(1-s \eta)^{2}} .
$$

Based on (3) and (4), the general form of the Mie-Gruneisen state equation is derived. In equation (4), the $c_{0}$ is the velocity of sound in water, and $s$ is the material parameter. Combined with the Hugoniot test data [38] of the water fluid impact, the fitting material parameters are calculated as shown in Figure 5(b). Thereinto, the constant $s$ is 1.92 and $\Gamma_{0}$ is equal to 1.2. The material parameters of the selected Mie-Gruneisen equation are $\rho_{0}=998.203 \mathrm{~kg} / \mathrm{m}^{3}$ and $c_{0}=1480 \mathrm{~m} / \mathrm{s}$.

To investigate the water flow characteristic in pores and the pore water pressure under moving traffic loads, the slight deformation of the tire and pavement is not considered. Therefore, the unidirectional fluid-solid coupling algorithm was adopted to ensure the calculation efficiency. The free surface of water flow was constructed on the fluid-solid coupling interface to transfer the pressure. The calculation was converged by repeated iterations, and the final hydrodynamic pressure value was obtained (as shown in Figure 6). Due to the large difference in the formation of the fluid mesh and the tire mesh, the weighted residual method was adopted to transfer and calculate the physical quantity between them [39]. In order to accelerate the calculation convergence, the water film in the calculation area was meshed, and the grids of contact surfaces were encrypted.
4.3. Setting ofBoundary Conditions. As for the water film inlet, a pressure inlet boundary is used to simulate the initial state of water film under wheel loading firstly. For the inlet boundary condition, the turbulent flow energy $\mathrm{K}$ and the fluid dissipation rate $\varepsilon$ are, respectively, defined according to (5 and 6).

$$
\begin{aligned}
K & =\frac{3}{2}(u I)^{2}, \\
\varepsilon & =\frac{0.09^{3 / 4} k^{3 / 2}}{l},
\end{aligned}
$$

where $\mu$ is the flow velocity, $l$ is the length of the water flow, and $I$ is the flow intensity.

As the vehicle drives over the asphalt pavement at high speed, it will cause splash of water flow. Thus, the water film surface also should be set as one of the exit boundaries. Both the water outlet and the upper surface of the water film are set as free flow to simulate the water splashing situation. It is assumed that the flow of water at the outlet is fully developed as incompressible fluid, and there is no backflow at the double boundary exit. The wall boundary condition was adopted for the bottom of the pavement model as well as the tread and the side of tire. Since the Reynolds stress near the wall disappears substantially inside the viscous underlayer and the turbulent motion gradually transforms into a laminar flow, it is necessary to realize the transition from laminar flow to turbulent flow through wall functions.

In order to ensure that the tire travels on actual slippery road surface, the fluid area covers the entire longitudinal road surface in simulation. Actually, when the tire rolls on the wet pavement, the water flow will splash due to the traffic loading. Therefore, an air layer with thickness of $50 \mathrm{~mm}$ was defined above the water film layer to ensure sufficient scattering space for water film splash when the tire rolls on the asphalt road surface. The moving and simple deforming objects were used to allow the tire move with a specific speed, which realize the coupling of the tire and the water flow.

4.4. Validation of Coupling Model. Set the water film thickness of $0 \mathrm{~mm}$ to simulate the tire rolling process, which is uniformly accelerated from $0 \mathrm{~km} / \mathrm{h}$ to $90 \mathrm{~km} / \mathrm{h}$ under dry road condition. Then, the vertical contact force was obtained and compared with wet pavement condition (as shown in Figure 7). It can be seen that the vertical contact force of the tire keeps around $3922 \mathrm{~N}$ on dry pavement. The main reason is that there is no dynamic water pressure in pores of pavement structure. As the 


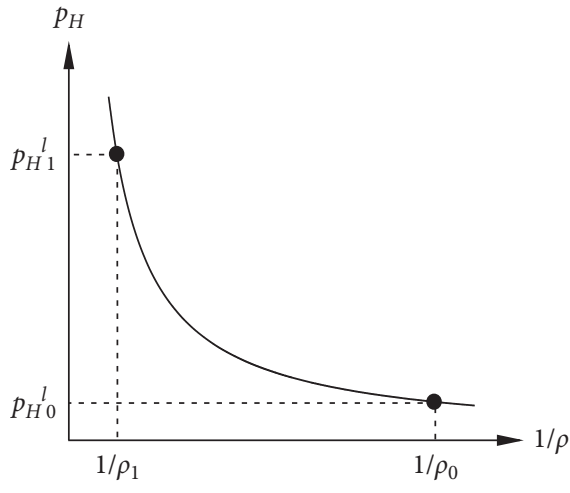

(a)

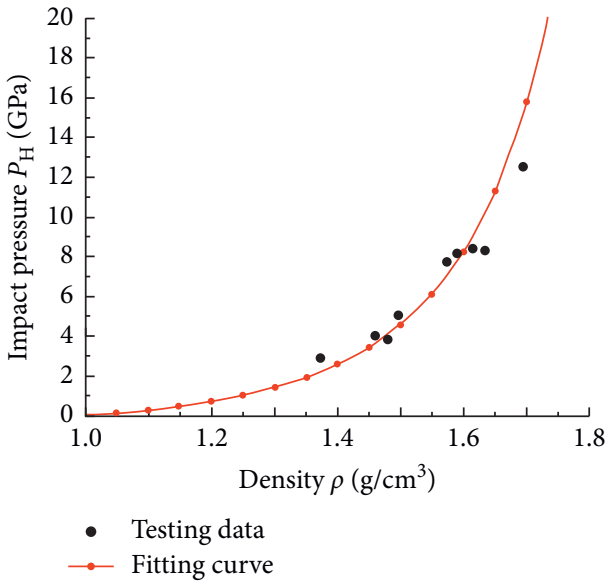

(b)

FIgURE 5: Hugoniot impact pressure curve of water flow: (a) standard Hugoniot impact curve; (b) fitting Hugoniot impact curve.

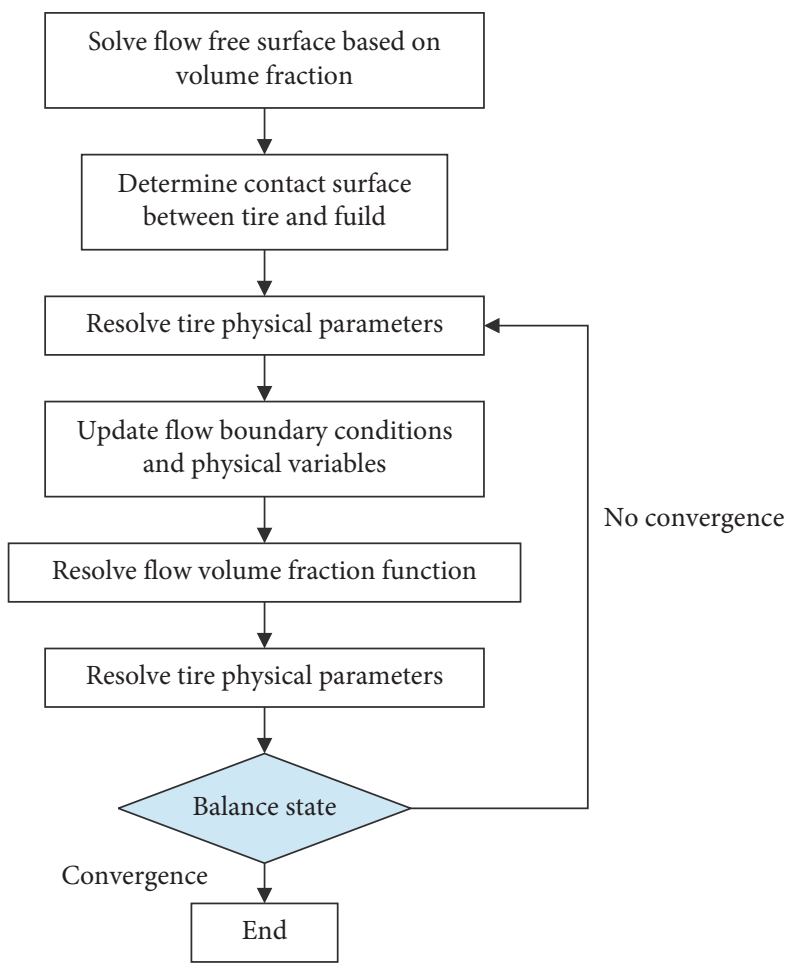

FIGURE 6: Contact algorithm of fluid-solid coupling model.

tire damping effect was not considered, the contact force of the tire exhibits slight fluctuation.

The lifting force of water flow to the tire was obtained according to the difference of vertical contact force between dry and wet road conditions as shown in Figure 7(a). Meanwhile, the relationship between the water flow lifting force and vehicle speed is shown in Figure 7(b). The slope of the fitted curve for water lifting force is approximately 2 . It indicates that the water lifting force is approximately proportional to the square of the vehicle speed, which is in accordance with reference [40]. Thus, it validates that the coupling model built in this study has certain accuracy.

\section{Calculation and Discussion}

5.1. Distribution Characteristics of Pore Water Pressure. Based on the established tire-fluid-road coupling model, the tire rolling speed and rotation speed were set as $60 \mathrm{~km} / \mathrm{h}$ and $8.40 \mathrm{r} / \mathrm{s}$, respectively. Also, the water film thickness was a constant of $5 \mathrm{~mm}$. The model was given an initial time step of 


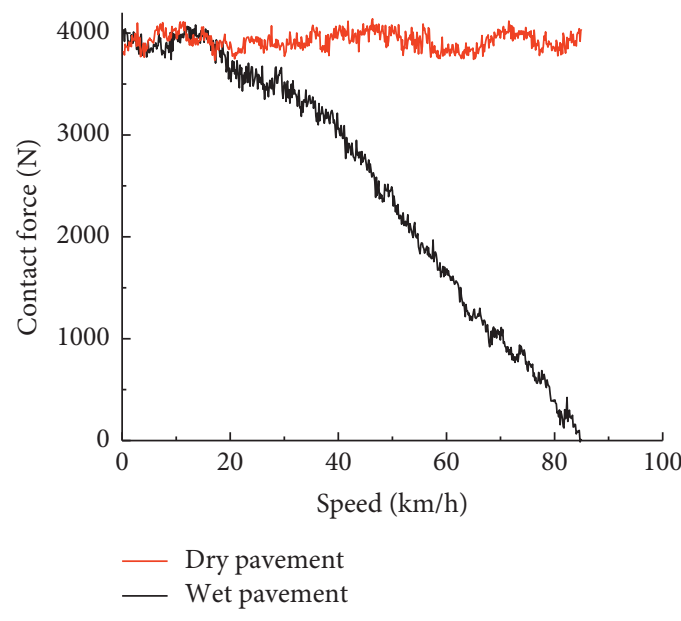

(a)

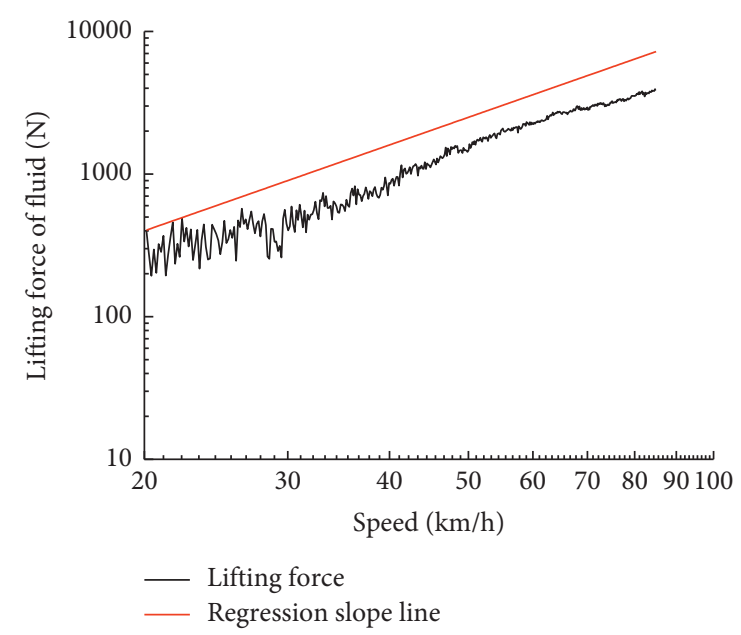

(b)

Figure 7: Comparison of vertical contact force on dry and wet roads. (a) Contact force curve of tire and pavement. (b) Fluid lifting force varying with speed.
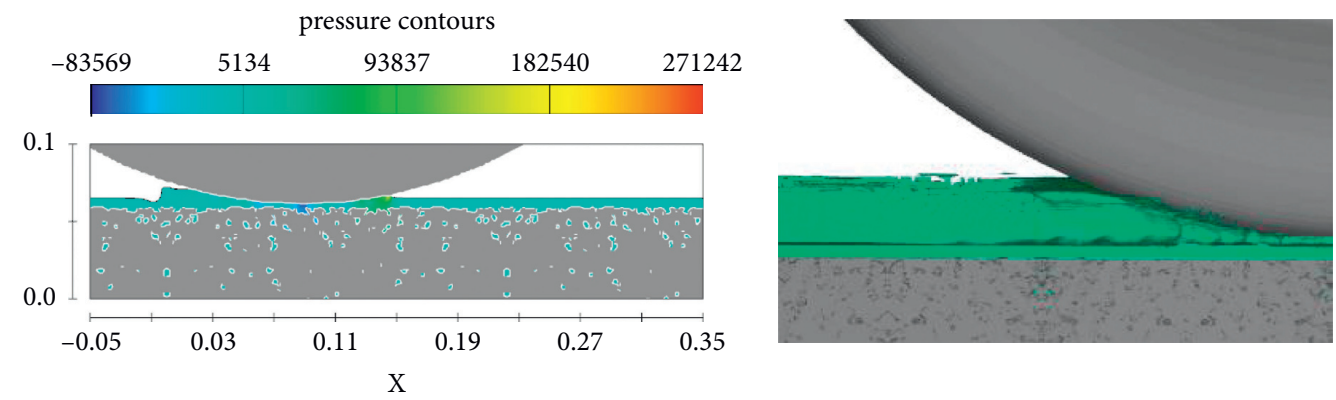

FIGURE 8: Simulation of pore water pressure distribution.

0.001 , and minimum time step is $1 \mathrm{e}-5$. The pressure-velocity coupled finite volume algorithm was applied to improve the calculation speed for model convergence. The distribution of pore water pressure within pavement structure was simulated as shown in Figure 8.

As the vehicle drives over the asphalt pavement at high speed, there are both positive pressure and negative pressure. Under the action of positive and negative alternating hydrodynamic pressures, asphalt mixture in pavement internal structure is peeled off, leading to certain moisture damage (such as holes, pumping, and so on) of the asphalt pavement.

The cube region was selected in the middle of the asphalt pavement to calculate the mean value of pore hydrodynamic pressure. The hydrodynamic pressure curve varying with time was obtained as shown in Figure 9. It can be seen that the pore water pressure in pavement internal structure under vehicle loading has a certain change regularity. The pore hydrodynamic pressure begins to appear at $14.81 \mathrm{~ms}$ and gradually increases with time. After reaching the peak value at $23.86 \mathrm{~ms}$, it begins to gradually decrease. After falling to $0 \mathrm{kPa}$, the hydrodynamic pressure starts to show a negative value and reaches the minimum value at $36.07 \mathrm{~ms}$. Then, it starts to gradually rise to zero, and there is a small fluctuation. Generally, the pore water pressure decays periodically over time, and this

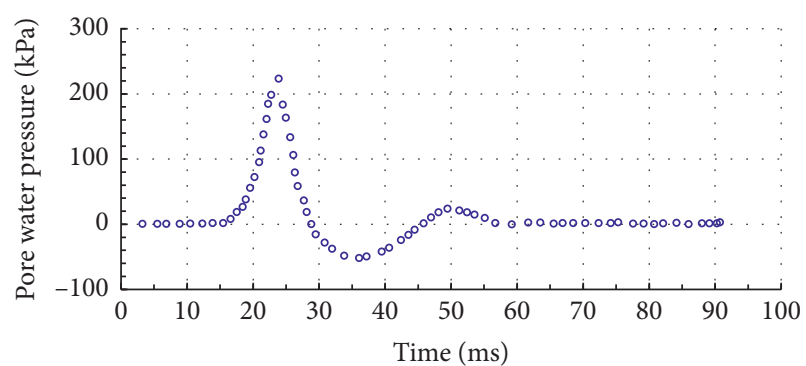

Figure 9: The pore water pressure curve varying with time.

phenomenon can be explained by hydrodynamic water hammer theory [41].

It can be seen that the pore hydrodynamic pressure periodically decays under traffic loading. This is because the built fluid model obtains the initial speed under the squeezing of high-speed rotating tire and the pavement. The internal free water produces a large impact force in pavement pores. The instantaneous impact force obtained by the water flow can be regarded as a pressure shock wave according to the fluid mechanics. Moreover, the energy will gradually dissipate during the water flow impact in pores. The pore water pressure in pavement structure gradually decreases as traffic loading moves away. 
TABLE 3: Gradations for three kinds of asphalt pavement.

\begin{tabular}{lcccccccccc}
\hline & \multicolumn{8}{c}{ Sieve size (mm) } \\
Pavement types & \multicolumn{8}{c}{ Passing rate of each sieve (\%) } \\
& 0.075 & 0.15 & 0.3 & 0.6 & 1.18 & 2.36 & 4.75 & 9.5 & 13.2 & 16 \\
\hline AC & 6 & 10 & 13.5 & 19 & 26.5 & 37 & 53 & 76.5 & 95 & 100 \\
SMA & 10 & 13.2 & 16.3 & 19.5 & 22.7 & 25.8 & 29 & 63.5 & 97.9 & 100 \\
OGFC & 4.6 & 5.4 & 6.1 & 8.7 & 11.5 & 15.0 & 18.8 & 63.3 & 97.8 & 100 \\
\hline
\end{tabular}

5.2. Influence of Pavement Type on Hydrodynamic Pressure. According to the above tire-fluid-pavement model, set the water film thickness as a constant of $5 \mathrm{~mm}$ with the driving speed of $60 \mathrm{~km} / \mathrm{h}$ and the wheel loading of $3500 \mathrm{~N}$. In this study, three kinds of asphalt pavement were, respectively, simulated with FLUENT 3D, dense-graded asphalt concrete (AC), stone matrix asphalt (SMA), and open-grade friction course (OGFC). The gradation design for the three asphalt mixtures is shown in Table 3 . The pore water pressure for different asphalt pavement is shown in Figure 10.

According to Figure 10, for the three types of asphalt pavement, the pore water pressure curves varying with time tend to be similar. When the pore water pressure changes from zero to positive peak and then returns to zero, the time spent is the same, that is to say, the type of asphalt pavement does not have much influence on the periodicity of pore hydrodynamic pressure. However, the pavement type has great influence on the positive peak value of the pore water pressure, while it has little influence on negative pressure (as shown in Table 4). Moreover, the negative peak values of pore water pressure are generally less than 0.3 times the positive peak values.

For the three types of pavements, the maximum values both of positive hydrodynamic pressure and negative hydrodynamic pressure are listed by AC pavement $>$ SMA pavement $>$ OGFC pavement. In addition, the pore water pressure of OGFC pavement is the smallest when the vehicle runs at high speed on rainy days. It can be seen that the OGFC pavement is superior to the other two types of asphalt pavement for preventing vehicle hydroplaning and reducing moisture damage.

\subsection{Influence of Water Film Thickness on Hydrodynamic} Pressure. Similarly, set the water film thickness of $1 \mathrm{~mm}$, $2 \mathrm{~mm}, 5 \mathrm{~mm}, 8 \mathrm{~mm}$, and $10 \mathrm{~mm}$, respectively, in the water film model (see Figure 11). AC asphalt pavement is taken as research object with constant tire speed of $60 \mathrm{~km} / \mathrm{h}$ and wheel loading of $3500 \mathrm{~N}$.

According to the simulation results, the average water hydrodynamic pressure in the calculated water region was analyzed. Moreover, the average hydrodynamic pressure curve varying with time under different water film thicknesses was obtained. It can be seen that under different water film thicknesses, the water hydrodynamic pressure values varying with time tend to be consistent. The maximum values of water hydrodynamic pressure with different water film thicknesses were obtained as shown in Figure 12.

It can be seen that the maximum negative value of hydrodynamic pressure increases slightly with the increasing

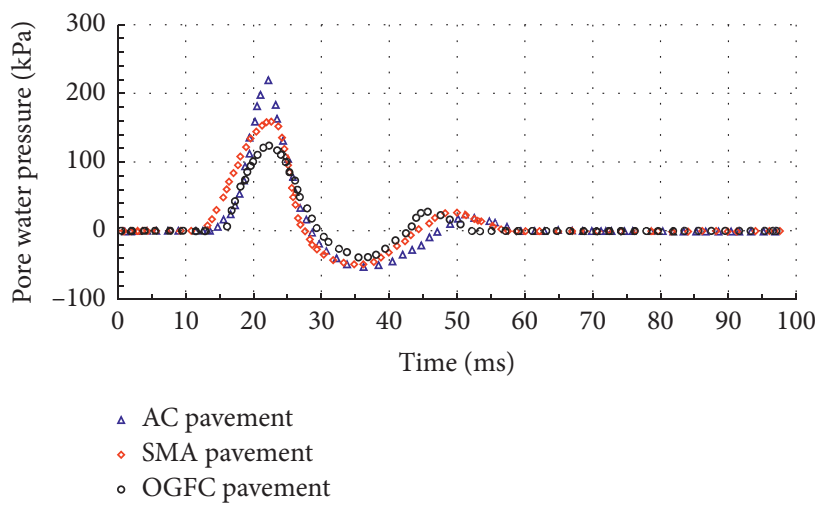

FIgURe 10: Pore water pressure varying with time for different kinds of asphalt pavement.

water film thickness. The maximum negative value of hydrodynamic pressure decreases by $13.02 \%$ as the water film thickness increases from $1 \mathrm{~mm}$ to $10 \mathrm{~mm}$. The maximum positive hydrodynamic pressure increases parabolically with increasing water film thickness. Compared with the influence of the water film thickness on the tire lifting force [42], the water film thickness has little effect on the hydrodynamic pressure. This is because the pores in pavement are usually saturated on rainy days. With same vehicle speed, the initial speed of water flow obtained by the tire squeezing is the same under different water film thicknesses. Therefore, the impact of different water film thickness on the asphalt pavement pore structure has no significant difference.

5.4. Influence of Tire Speed on Hydrodynamic Pressure. In order to study the influence of driving speed on the pore hydrodynamic pressure in asphalt pavement, keeping the water film thickness and wheel loading constant of $5 \mathrm{~mm}$ and $3500 \mathrm{~N}$, respectively, the driving speed is, respectively, selected to be $30 \mathrm{~km} / \mathrm{h}, 60 \mathrm{~km} / \mathrm{h}, 90 \mathrm{~km} / \mathrm{h}, 100 \mathrm{~km} / \mathrm{h}$, and $120 \mathrm{~km} / \mathrm{h}$. Through simulation results, the pore hydrodynamic pressure in asphalt pavement at different vehicle speeds was obtained.

The hydrodynamic pressure varying with time in asphalt pavement at different vehicle speeds has a consistent trend. Firstly, the hydrodynamic pressure increases to a peak value and then decreases with time, until dropping to the maximum negative value. It gradually increases from the maximum negative value to 0 . Then, it shows a small range of fluctuations until it becomes 0 finally. As the tire gradually rolls away from the calculation area, the hydrodynamic pressure gradually becomes 0 . On the whole, the 
TABLE 4: Peak values of pore water pressure in pavement structure.

\begin{tabular}{lccc}
\hline Items & & Asphalt pavement types \\
SMA pavement & PC pavement & 159.38 & 124.27 \\
\hline Maximum positive $(\mathrm{kPa})$ & 219.31 & -48.19 & -37.11 \\
Maximum negative $(\mathrm{kPa})$ & -56.88 & 29.61 & 29.86 \\
Relative change rate $(\%)$ & 25.94 & OGFC & 29.8 \\
\hline
\end{tabular}

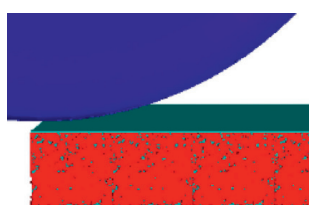

(a)

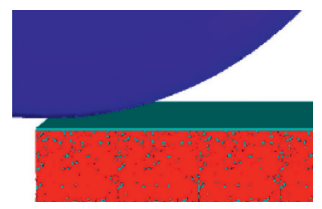

(b)

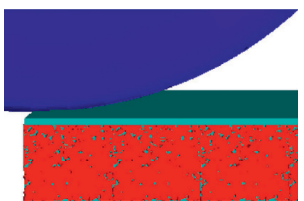

(c)

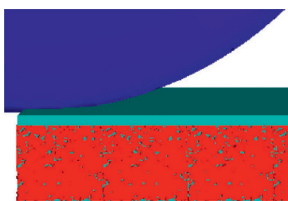

(d)

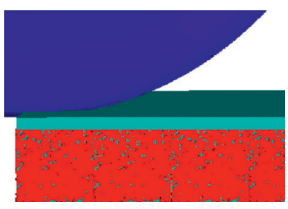

(e)

Figure 11: Water film thickness: (a) $1 \mathrm{~mm}$; (b) $2 \mathrm{~mm}$; (c) $5 \mathrm{~mm}$; (d) $8 \mathrm{~mm}$; (e) $10 \mathrm{~mm}$.

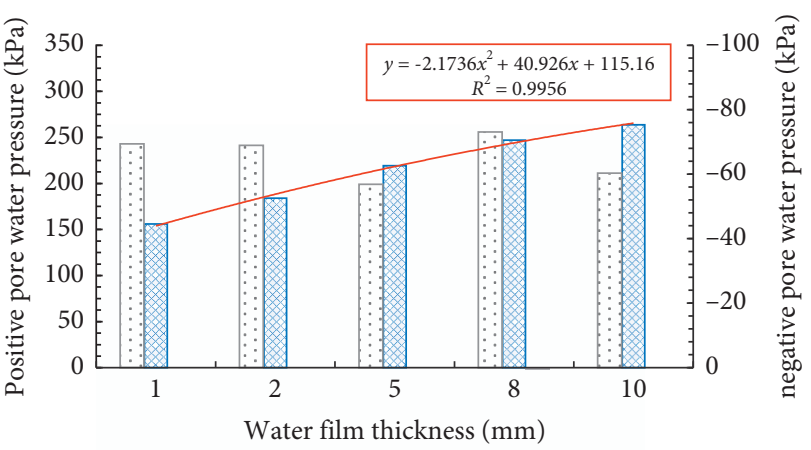

๑ Maximum positive value

๖ Maximum negative value

FIGURE 12: Maximum hydrodynamic pressure with different water film thicknesses.

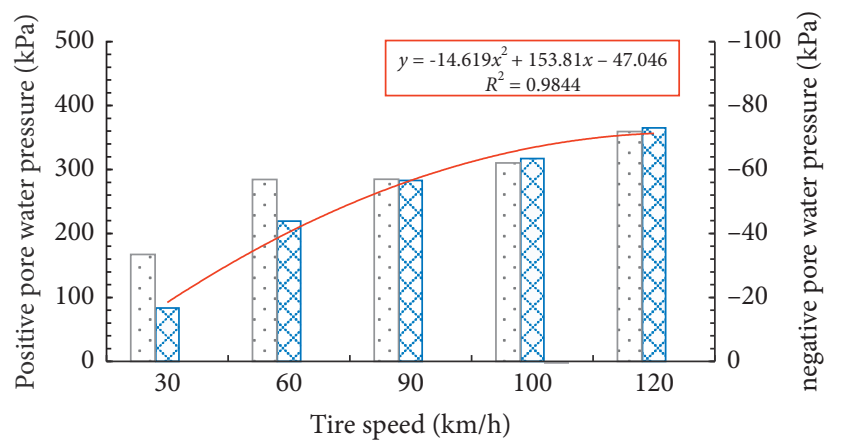

- Maximum positive value

$\square$ Maximum negative value

FIGURE 13: Maximum hydrodynamic pressure at different tire speeds.

hydrodynamic pressure in asphalt pavement appears periodically and decays constantly with the increasing tire speed.

According to the pore water pressure curves varying with time, the peak value of pore water pressure at different speeds was obtained (as shown in Figure 13). It can be seen that the positive peak values of hydrodynamic pressure increase parabolically with increasing driving speed.
However, the negative peak values change little with vehicle speed. As the vehicle speed increases from $30 \mathrm{~km} / \mathrm{h}$ to $120 \mathrm{~km} / \mathrm{h}$, the maximum positive value of pore water pressure increases by $281.60 \mathrm{kPa}$, while the maximum negative value just increases by $38.41 \mathrm{kPa}$. The higher the vehicle speed is, the shorter the time needed for the pore water pressure to reach the peak value, that is, the shorter the periodicity of the pore water pressure variation is.

\section{Summary and Conclusions}

To investigate the variation rule of pore water pressure in asphalt pavement under vehicle loading, a novel method based on BA network and QSGS method for the reconstruction of asphalt pavement 3D model with pores was proposed. The permeability coefficient and the gradation curve were used to validate the reliability of the random growth pavement model. Then, based on the fluid MieGruneisen state equation, the tire-water-pavement coupling model was established by FLUENT 3D to analyze the distribution characteristics of pore water pressure under vehicle loading. The main findings are as follows:

(1) Based on BA network and QSGS method, the established random growth model of asphalt pavement is close to the formation process of porous media. It has a fast growth rate and a uniform void distribution. Meanwhile, compared with the randomly generated model method with CT technology, the largest relative error is $2.62 \%$, which is within the range of allowable error of $5 \%$.

(2) When the vehicle drives at high speed under wet road condition, the pore water pressure in pavement internal structure has a certain periodicity and attenuation, which can be explained by hydrodynamic water hammer theory. Under the repeated extrusion of positive and negative hydrodynamic pressures, the asphalt mixtures easily peel off and further result in moisture damage of asphalt pavement.

(3) The change rule of the pore water pressure with different pavement types tends to be consistent 
with time. There is no obvious influence of pavement type on the periodicity of the pore water pressure. The peak value of pore water pressure in AC pavement is the largest, followed by SMA pavement and OGFC pavement. The maximum negative value of the pore water pressure is generally less than 0.3 times the maximum positive values.

(4) The maximum negative value of the pore water pressure increases slightly as the thickness of the water film increases, while the maximum positive pore water pressure increases parabolically with the increase of the thickness of the water film. As the water film thickness increases from $1 \mathrm{~mm}$ to $10 \mathrm{~mm}$, the maximum negative value of pore water pressure decreases by $13.02 \%$. When the pavement pores are already saturated, the thickness of the water film has little effect on the pore water pressure.

(5) Under different vehicle speeds, the pore water pressure appears to be periodic and attenuating. As the vehicle speed improves from $30 \mathrm{~km} / \mathrm{h}$ to $120 \mathrm{~km} /$ $\mathrm{h}$, the maximum positive value increases by $281.60 \mathrm{kPa}$ while the maximum negative value of pore water pressure only increases by $38.41 \mathrm{kPa}$. The larger the tire speed, the shorter the variation periodicity of the pore water pressure.

Since the pavement model with void and texture is too complex, the tread pattern was simplified in the tire-fluidpavement model in this study. The subsequent consideration should be given to the water flow characteristic for different tread groove types when the vehicle drives on wet road surface at high speed.

\section{Data Availability}

Previously reported data were used to support this study and are available at DOI: $10.1155 / 2017 / 5843061$. These prior studies (and datasets) are cited at relevant places within the text as references $[34,36,37]$.

\section{Conflicts of Interest}

The authors declare that they have no conflicts of interest.

\section{Authors' Contributions}

The work presented here was carried out in collaboration between all authors. Binshuang Zheng was responsible for study conception and design. Jingwen Ma and Jiaying Chen were responsible for data collection. Jiaying Chen and Runmin Zhao were responsible for analysis and interpretation of results. Shengze Zhu was responsible for software. Zhengqiang Hong was responsible for draft manuscript review. Xiaoming Huang was responsible for funding acquisition and project administration. All authors reviewed the results and approved the final version of the manuscript.

\section{Acknowledgments}

This study was financially supported by National Natural Science Foundation of China (no. 51778139) and Natural Science Research Start-Up Foundation of Recruiting Talents of Nanjing University of Posts and Telecommunications (no. NY221150).

\section{References}

[1] A. K. Apeagyei, J. R. A. Grenfell, and G. D. Airey, "Evaluation of moisture sorption and diffusion characteristics of asphalt mastics using manual and automated gravimetric sorption techniques," Journal of Materials in Civil Engineering, vol. 26, no. 8, Article ID 04014045, 2014.

[2] R. A. Tarefder and M. Ahmad, "Evaluating the relationship between permeability and moisture damage of asphalt concrete pavements," Journal of Materials in Civil Engineering, vol. 27, no. 5, Article ID 04014172, 2015.

[3] Z. J. Gao, X. Z. Cui, W. Sui et al., "Analysis of influence factors of drainage effect for porous pavement based on unsaturated seepage theory," Highways, vol. 10, pp. 70-74, 2014.

[4] S. B. Li, H. B. Zhang, and L. J. Sun, "Development and simulation measurement of dynamic hydraulic pressure," Journal of Tongji University, vol. 35, no. 7, pp. 915-918, 2007.

[5] Y. X. Cai, A. Q. Shen, Y. C. Guo, and D. Q. Wang, "Dynamic water damage behavior and dynamic seepage test for asphalt mixture," Journal of Chang'an University (Natural Science Edition), vol. 35, no. 2, pp. 13-25, 2015.

[6] S. H. Kim, J. H. Jeong, and N. Kim, "Use of surface free energy properties to predict moisture damage potential of asphalt concrete mixture in cyclic loading condition," KSCE Journal of Civil Engineering, vol. 7, no. 4, pp. 381-387, 2003.

[7] Q. Xue and L. Liu, "Hydraulic-stress coupling effects on dynamic behavior of asphalt pavement structure material," Construction and Building Materials, vol. 43, no. 3, pp. 31-36, 2013.

[8] Z. G. Li and X. Y. Deng, "Axial symmetric elastic solution of pore water pressure in asphalt pavement under mobile load," Journal of Southeast University (Natural Science Edition), vol. 38, no. 5, pp. 804-810, 2008.

[9] J. Huang, Y. Cai, S. B. Dai, and X. Pan, "Dynamic response of saturated asphalt pavement under vehicle loading," in Proceedings of the 2015 4th International Conference On Computer, Mechatronics, Control And Electronic Engineering (ICCMCEE 2015), Series: Advances in Engineering Research, pp. 338-343, Hangzhou, China, November 2015.

[10] X. Guo, M. Sun, and W. Dai, "Analysis of effective pore pressure in asphalt pavement based on computational fluid dynamics calculation," Advances in Mechanical Engineering, vol. 9, no. 2, pp. 1-14, 2017.

[11] W. T. Wang, L. B. Wang, H. C. Xiong, and R. Luo, “A review and perspective for research on moisture damage in asphalt pavement induced by dynamic pore water pressure," Construction and Building Materials, vol. 204, pp. 631-642, 2019.

[12] W. X. Chen, Z. Y. Wang, W. Guo, and W. Dai, "Measurement and evaluation for interbedded pore water pressure of saturated asphalt pavement under vehicle loading," Applied Sciences, vol. 10, no. 4, p. 13, Article ID 1416, 2020.

[13] C. D. Si, E. L. Chen, Z. P. You, R. Zhang, P. Qiao, and Y. Feng, "Dynamic response of temperature-seepage-stress coupling in asphalt pavement," Construction and Building Materials, vol. 211, pp. 824-836, 2019. 
[14] Y. Z. Sun, R. Guo, L. Gao, J. Wang, X. Wang, and X. Yuan, "Study on dynamic response characteristics of saturated asphalt pavement under multi-field coupling," Materials, vol. 12, no. 6, p. 15, Article ID 959, 2019.

[15] J. Q. Gao, C. C. Guo, and Y. T. Liu, "Measurement of pore water pressure in asphalt pavement and its effects on permeability," Measurement, vol. 62, no. 4, pp. 81-87, 2015.

[16] E. Arambula, S. Caro, and E. Masad, "Experimental measurement and numerical simulation of water vapor diffusion through asphalt pavement materials," Journal of Materials in Civil Engineering, vol. 22, no. 6, pp. 588-598, 2010.

[17] P. Kettil, M. G. Engstr, and N. E. Wiberg, "Coupled hydromechanical wave propagation in road structures," Computers \& Structures, vol. 83, no. 21-22, pp. 1719-1729, 2005.

[18] A. Bozorgzad, S. F. Kazemi, and F. M. Nejad, "Evaporationinduced moisture damage of asphalt mixtures: microscale model and laboratory validation," Construction and Building Materials, vol. 171, pp. 697-707, 2018.

[19] M. T. Weldegiorgis and R. A. Tarefder, "Towards a mechanistic understanding of moisture damage in asphalt concrete," Journal of Materials in Civil Engineering, vol. 27, no. 3, Article ID 04014128, 2015.

[20] I. M. A. Abuawad, I. L. Al-Qadi, and J. S. Trepanier, "Mitigation of moisture damage in asphalt concrete: testing techniques and additives/modifiers effectiveness," Construction and Building Materials, vol. 84, pp. 437-443, 2015.

[21] H. Min, W. Zhang, and X. Gu, "Effects of load damage on moisture transport and relative humidity response in concrete," Construction and Building Materials, vol. 169, pp. 59-68, 2018.

[22] H. C. Dan, L. H. He, L. H. Zhao, and J. Chen, "Coupled hydromechanical response of saturated asphalt pavement under moving traffic loading," International Journal of Pavement Engineering, vol. 16, no. 2, pp. 125-143, 2015.

[23] W. H. Jiang, X. N. Zhang, and Z. Li, "Mechanical mechanism of moisture-induced damage of asphalt mixture based on simulation test of dynamic water pressure," China Journal of Highway and Transport, vol. 24, no. 4, pp. 21-25, 2011.

[24] Y. Y. Liu, Y. Y. Wang, X. Y. Cai, and X. Hu, "The detection effect of pavement 3D texture morphology using improved binocular reconstruction algorithm with laser line constraint," Measurement, vol. 157, p. 9, Article ID 107638, 2020.

[25] H. Wang, Z. Huang, L. Li, Z. Yu, and Y. Chen, "Three-dimensional modeling and simulation of asphalt concrete mixtures based on X-ray CT microstructure images," Journal of Traffic and Transportation Engineering, vol. 1, no. 1, pp. 55-61, 2014.

[26] M. E. Kutay, E. Arambula, N. Gibson, and J. Youtcheff, "Three-dimensional image processing methods to identify and characterise aggregates in compacted asphalt mixtures," International Journal of Pavement Engineering, vol. 11, no. 6, pp. 511-528, 2010.

[27] M. Ran, J. Zhang, X. Cui, and X. Zhou, X. Cui, Depth-fromfocus-based $3 \mathrm{D}$ reconstruction of asphalt pavement microtexture," Journal of Testing and Evaluation, vol. 46, no. 1, pp. 419-427, 2017.

[28] Y. Wang, Q. Dong, and Y. Chen, "Seepage simulation using pipe network flow model in a discrete element system," Computers and Geotechnics, vol. 92, pp. 201-209, 2017.

[29] C. H. Zhou, J. Y. Chen, Z. R. Wang et al., "Dynamic numerical method of pore water pressure and its influence parameters for asphalt pavement," Journal of Central South University, vol. 87, no. 11, pp. 1615-1618, 2008.
[30] F. Q. Zhang, X. Q. Teng, and B. B. Li, "Meso mesoscopic model of asphalt concrete based on random aggregate placement," Journal of Railway Science and Engineering, vol. 16, no. 5, pp. 1216-1222, 2019.

[31] M. Wang, J. Wang, N. Pan et al., "Mesoscopic predictions of the effective thermal conductivity for microscale random porous media," Physical Review E, vol. 75, no. 3, Article ID 036702, 2007.

[32] R. M. Li, S. Y. Liu, L. Fang, and Y. J. Du, "Micro-structure of clay generated by quartet structure generation set," Journal of Zhejiang University, vol. 44, no. 10, pp. 1897-1901, 2010.

[33] X. C. Geng and W. Chen, "Scale free network model based on total degree of adjacent nodes and random division," Software Guide, vol. 17, no. 6, pp. 190-193, 2018.

[34] B. S. Zheng, J. Y. Chen, R. M. Zhao et al., "Analysis of contact behaviour on patterned tire-asphalt pavement with 3-D FEM contact model," International Journal of Pavement Engineering, vol. 2020, no. 6, pp. 1-16, 2020.

[35] B. N. J. Persson, "Theory of rubber friction and contact mechanics," The Journal of Chemical Physics, vol. 115, no. 8, pp. 3840-3861, 2001.

[36] S. Z. Zhu, X. Y. Liu, Q. Q. Cao, and X. Huang, "Numerical study of tire hydroplaning based on power spectrum of asphalt pavement and kinetic friction coefficient," Advances in Materials Science and Engineering, vol. 2017, p. 11, Article ID 5843061, 2017.

[37] S. Z. Zhu and X. M. Huang, "Numerical simulation of tire critical hydroplaning speed on transverse grooved concrete pavement," Journal of Southeast University (Natural Science Edition), vol. 46, no. 6, pp. 1296-1300, 2016.

[38] K. Anupam, "Numerical simulation of vehicle hydroplaning and skid resistance on grooved pavement," $\mathrm{Ph}$. D thesis, National University of Singapore, Singapore, Singapore, 2012.

[39] A. de Boer, A. H. V, and H. Bijl, "Review of coupling methods for non-matching meshes," Computer Methods in Applied Mechanics and Engineering, vol. 196, no. 8, pp. 1515-1525, 2007.

[40] E. Seta, Y. Nakajima, T. Kamegawa, and H. Ogawa, "Hydroplaning analysis by FEM and FVM: effect of tire rolling and tire pattern on hydroplaning," Tire Science and Technology, vol. 28, no. 3, pp. 140-156, 2000.

[41] S. R. Wang, T. W. Liu, and T. G. Peng, Water Hammer Theory and Water Hammer Calculation, Tsinghua University Press, Beijing, China, 1980.

[42] X. M. Huang, Q. Q. Cao, X. Y. Liu, J. Y. Chen, and X. L. Zhou, "Simulation of vehicle braking performance on rainy days based on pavement surface fractal friction theory," Journal of Jilin University (Engineering and Technology Edition), vol. 49, no. 3, pp. 757-765, 2019. 